\title{
ANÁLISE COMPARATIVA ENTRE DUAS ESCALAS DE AVALIAÇÃO CLÍNICA NA ESCLEROSE MÚLTIPLA
}

\author{
REVISÃO DE 302 CASOS \\ EDUARDO FELIPE*, MARIA FERNANDA MENDES*, \\ MARCO AURÉLIO MOREIRA **, CHARLES PETER TILBERY ${ }^{* * *}$
}

RESUMO - Muitas escalas neurológicas têm sido utilizadas para avaliar evolutivamente portadores da esclerose múltipla. O Expanded Disability Status Scale (EDSS) de Kurtzke ainda é a mais utilizada. Analisamos comparativamente a aplicação do EDSS e Neurologic Rating Scale (NRS), de Sipe e col. em 302 pacientes com forma definida de esclerose múltipla. Os resultados demonstram que o NRS foi mais sensível que o EDSS na detecção de alterações clínicas em 22,1\% dos casos, sendo possivelmente mais sensível na caracterização de um novo surto. As variações do EDSS ocorrereram mais nos pacientes com EDSS de 36,0 e 3,5. Fazemos considerações sobre estes achados, sugerindo a aplicação de mais de uma escala clínica para avaliação dos pacientes nos estudos com novas drogas para tratamento da doença.

PALAVRAS-CHAVE: esclerose múltipla, escalas de avaliação clínica, EDSS, NRS.

\section{Comparative analysis of two clinical scales for multiple sclerosis: review of $\mathbf{3 0 2}$ cases}

ABSTRACT - Many neurologic scales have been used for clinical evaluation of multiple sclerosis, but there is no consensus about which one is the most appropriate to assess evolution and point to a new relapse. The Expanded Disability Status Scale (EDSS) has been the most commonly used. We analyse the reliability of two scales: the EDSS and Neurologic Rating Scale (NRS) in 302 multiple sclerosis patients. It is shown that NRS is a more sensitive scale than EDSS to disclose clinical changes (22.1\% of cases). Changes in NRS were more evident in patients with EDSS 3.0 and 3.5. We comment on these findings and suggest that both scales should be employed in multiple sclerosis treatment trials.

KEY WORDS: multiple sclerosis, clinical evaluation scales, EDSS, NRS.

Desde 1993, tem-se demonstrado benefício no uso do interferon beta e do acetato de glatiramer na esclerose múltipla, resultado demonstrado em várias publicações de estudos multicêntricos, em que se observou redução das lesões ativas na ressonância nuclear magnética do crânio e redução do número de $\operatorname{surtos}^{1-4}$. As duas escalas clínicas mais utilizadas nestes estudos são o Expanded Disability Status Scale (EDSS) e o Neurologic Rating Scale (NRS).

O EDSS é a escala mais difundida para avaliação da esclerose múltipla. Possui vinte itens com escores variando de 0 a 10 , com pontuação aumentando em meio ponto conforme o grau de

Estudo da Disciplina de Neurologia do Departamento de Medicina da Santa Casa de Misericórdia de São Paulo, Centro de Atendimento e Tratamento da Esclerose Múltipla (CATEM): * Instrutor de Ensino da Disciplina de Neurologia e do Pronto Socorro da Santa Casa de São Paulo, ** Neurologista, *** Professor Adjunto da Disciplina de Neurologia. Aceite: 17-janeiro-2000.

Dr. Charles Peter Tilbery - Av. Albert Einstein, 627/1217 - 05652-901 São Paulo SP - Brasil. Fax 1130643332. E-mail edfelipe@zaz.com.br. 
incapacidade do paciente, dando maior enfoque à capacidade de deambulação do paciente (principalmente nas pontuações acima de 4,0), apoiada por escala de avaliação funcional de sistemas(FS). ${ }^{5}$

O NRS é escala de 100 pontos, baseada nos achados do exame neurológico, em que a redução dos pontos indica um maior comprometimento clínico do paciente. Para cada sintoma ou sinal clínico abordado nesta escala, a pontuação varia de acordo com comprometimento leve, moderado ou grave, que é definido por uma avaliação subjetiva do examinador que dá uma pontuação variando de $-4 \mathrm{a}+4$.

O EDSS geralmente é utilizado em grandes estudos multicêntricos para estadiar o grau de incapacidade do paciente. O NRS é utilizado mais para quantificar a intensidade de um surto. Os critérios de Schumacher para diagnóstico de um novo surto (surgimento de um novo ou antigo sintoma ou sinal neurológico com duração mínima de 24 horas) têm sido utilizados nestes estudos; entretanto, tem-se observado também a utilização do EDSS como critério de definição de surto.

Baseados nas diferentes metodologias apresentadas para comprovar eficácia de uma nova terapêutica para esclerose múltipla e dando principal enfoque à redução do número de surtos, comparamos o EDSS e o NRS de 302 pacientes, que seguem em acompanhamento clínico no Centro de Atendimento e Tratamento da Esclerose Múltipla (CATEM), com o objetivo de determinar qual destas escalas possui maior sensibilidade na detecção de alterações clínicas, portanto maior eficácia na detecção de novos surtos.

\section{MÉTODO}

Foram selecionados os escores do EDSS e NRS da primeira e última avaliações de 302 pacientes que seguem em tratamento no CATEM desde dezembro de 1996 até julho de 1998, todos apoiados por ressonância nuclear magnética e líquido cefalorraquidiano com pesquisa de eletroforese de proteínas e dosagem de IgG. Todos os pacientes foram submetidos ao exame neurológico, EDSS e NRS. Por se tratar de trabalho retrospectivo, foram considerados os escores do EDSS e NRS obtidos anteriormente a este período.

A média de idade dos pacientes no final da pesquisa foi 37,7 anos, com relação de três mulheres para um homem. A idade média de início da sintomatologia foi de 29,6 anos com um período médio de início da sintomatologia de 8 anos, e variação entre 2 meses e 27 anos.

O período de assistência médica variou de 02 meses a 17 anos, com média de 2,5 anos.

Duzentos e vinte casos apresentavam forma remitente-recorrente, 41 casos forma primariamente progressiva e 41 a forma secundariamente progressiva. Sessenta e três casos foram submetidos à primeira e última avaliações por neurologistas distintos e foram excluídos da análise comparativa para evitar uma variabilidade entre examinadores na aplicação das escalas. Dezenove casos foram excluídos por não apresentarem o NRS inicial.

O intervalo mínimo entre a primeira e a última avaliação foi de um mês e o máximo de 108 meses, com variação média de 30 meses.

As variações do NRS e EDSS foram analisadas estatisticamente pela técnica do qui-quadrado.

\section{RESULTADOS}

Foram analisados os resultados de 220 pacientes do total de 302 . Somente 39 casos apresentavam NRS e EDSS estáveis. O intervalo entre a primeira e última avaliações neste grupo variou entre um e cinco meses. Cento e oitenta e um pacientes apresentaram variações nos escores finais do EDSS e NRS comparados aos iniciais.

Todos os pacientes apresentaram variação no NRS (100\%) e apenas 141 no EDSS $(77,9 \%)$. Nenhum dos pacientes analisados apresentou variação no EDSS com NRS estável. O NRS foi mais sensível em $22,1 \%$ nesta amostra, na detecção de alterações clínicas.

Analisando-se os 41 pacientes, 15 apresentavam EDSS de 3,5; nove pacientes escore de 3,0; quatro com escores de 2,0; quatro com escore de 1,5. O restante dos escores obtidos está discriminado no gráfico (Figura). 


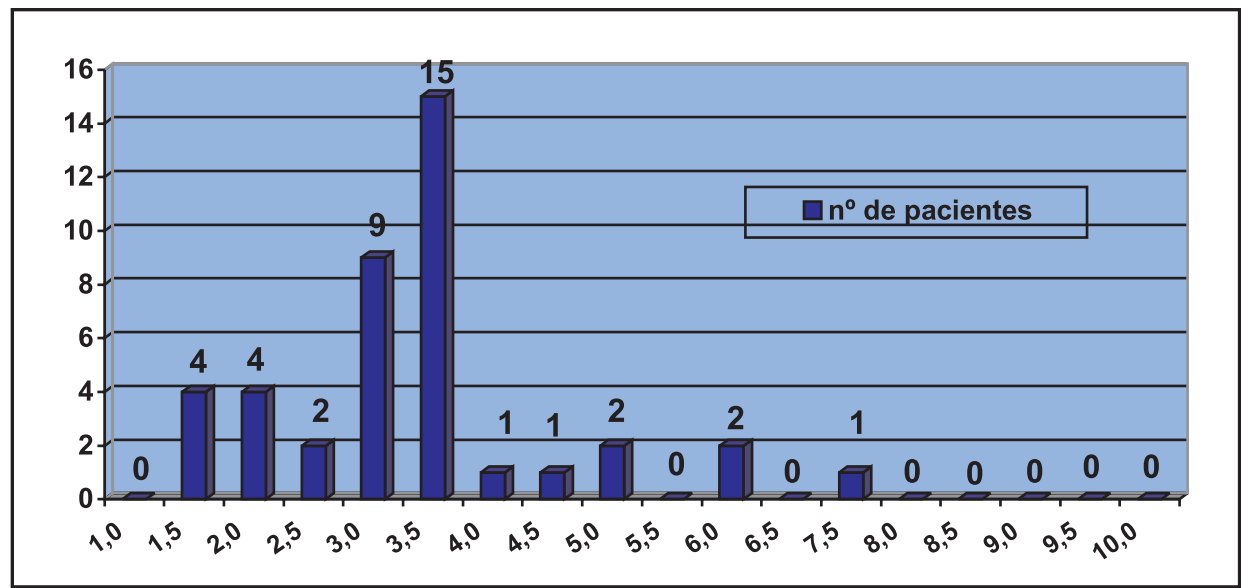

Figura. Distribuição dos 41 pacientes portadores de esclerose múltipla com EDSS estável e variação no NRS.

\section{DISCUSSÃO}

As escalas abordadas neste estudo são de grande complexidade, podendo apresentar diferenças de escores finais em um paciente examinado por dois neurologistas distintos. Esta variabilidade tem sido descrita na literatura, Sipe e col. ${ }^{6}$ descrevem variabilidade entre examinadores de até 10 pontos do NRS com coeficiente Kappa de 0,828. Entretanto, não se encontram na literatura dados semelhantes e a diferença de 10 pontos é muito significativa na avaliação da intensidade de um surto. O coeficiente Kappa, para um examinador apenas, é descrito como variação entre 0,978 e 0,998.

O EDSS possui variabilidade de escore para um examinador apenas entre 0,876 e 0,961, muito semelhante à já descrita anteriormente para o NRS.

Levando-se em consideração apenas pacientes vistos em uma primeira e última avaliações por um mesmo neurologista, atingimos o objetivo de obter um resultado estatisticamente mais fiel, visto que os índices Kappa para ambas as escalas aplicadas por um único examinador tem valores maiores que 0,81 .

Apesar da caracterização do NRS como 22,1\% mais sensível que o EDSS, deve-se levar em consideração o fato de serem dados retrospectivos em que não foi possível a omissão dos escores da primeira avaliação ao examinador, antes de realizar a última avaliação, o que não garante uma possível influência na aplicação das escalas e realização do exame neurológico, sobretudo quando as avaliações são separadas por um período muito curto de tempo. Esse detalhe pode ter contribuído para que 39 pacientes $(17,7 \%)$ apresentassem o mesmo escore do EDSS e do NRS em um intervalo de tempo variando apenas entre um e cinco meses.

Em quatro estudos multicêntricos envolvendo as principais opções de terapêutica atuais: interferon-beta 1-a, interferon-beta 1-b e acetato de glatiramer, tem-se observado diferentes metodologias para definição de surto e avaliação da intensidade de surto, como demonstrado da Tabela.

Os critérios adotados pelo INFB Group são semelhantes, adotados os critérios de Schumacher para definição de um novo surto, não se utilizando de qualquer escala clínica para caracterizar o surto, entretanto, dividindo em surtos leves, moderados ou graves conforme o decréscimo no NRS (respectivamente pontuação de 0 a $7 ; 7$ a 15 ; maior que 15).

Já os critérios de Johnson e col. ${ }^{3}$ e Jacobs e col. ${ }^{4}$, consideram a ocorrência de novo surto apenas quando observa-se aumento de meio ponto no EDSS ou um ponto em dois sistemas funcionais de Kurtzke, o que pode, pelos resultados apresentados nesta revisão, representar menor sensibilidade na detecção de surtos, assim comprometendo a afirmação de que essas drogas estudadas reduziriam o número de surtos dos pacientes ${ }^{7-9}$. 
Tabela. Definição e avaliação da intensidade de surtos em diferentes estudos terapêuticos multicêntricos.

\begin{tabular}{|c|c|c|c|}
\hline $\begin{array}{c}\text { Estudo } \\
\text { multicêntrico }\end{array}$ & $\begin{array}{l}\text { Critérios para } \\
\text { definir surto }\end{array}$ & $\begin{array}{l}\text { Escalas para } \\
\text { definir surto }\end{array}$ & $\begin{array}{c}\text { Avaliação da } \\
\text { intensidade de surto }\end{array}$ \\
\hline Infbeta group & Schumacher e col. & Nenhuma & NRS \\
\hline Jacobs e col. & $\begin{array}{l}\text { Sintoma ou sinal clínico novo ou } \\
\text { preexistente com duração mínima } \\
\text { de } 48 \text { horas e máxima de } 30 \text { dias. } \\
\text { Aumento em meio ponto no } \\
\text { EDSS ou um ponto da FS. }\end{array}$ & EDSS e FS & Nenhuma \\
\hline Prisms group & Schumacher e cols. & Nenhuma & NRS \\
\hline Johnson e col. & $\begin{array}{l}\text { Sintoma ou sinal clínico novo ou } \\
\text { preexistente com duração mínima } \\
\text { de } 48 \text { horas e máxima de } 30 \text { dias. } \\
\text { Aumento em meio ponto no } \\
\text { EDSS ou um ponto da FS }\end{array}$ & EDSS e FS & Nenhuma \\
\hline
\end{tabular}

Sipe e col. ${ }^{6}$ dão ênfase em sua publicação a maior sensibilidade do NRS, por refletir e pontuar as alterações do exame neurológico. Demonstram a aplicação da escala em seis pacientes em que se pode observar variações na pontuação do NRS com estabilidade do EDSS.

Podemos concluir por esta análise retrospectiva que o NRS foi mais sensível que o EDSS na detecção de alterações clínicas, portanto, corroborando os dados publicados por Sipe e col., de ser mais efetiva na detecção e avaliação da intensidade dos surtos. O EDSS, que avalia melhor a capacidade de deambulação, não deve ser utilizado como critério de definição e quantificação dos surtos. Ambas as escalas devem ser utilizadas em conjunto para estudos multicêntricos futuros.

\section{REFERÊNCIAS}

1. The INFB Multiple Sclerosis Study Group and the University of British Columbia MS/MRI Analysis Group. Interferon beta-1b in the treatment of multiple sclerosis: final outcome of the randomized controlled trial. Neurology 1995;45:12771285 .

2. PRISMS (Prevention of Relapses and disability by Interferon beta 1 a Subcutaneously in Multiple Sclerosis) Study Group. Randomised double-blind placebo-controlled study of interferon beta 1 a in relapsing/remiting multiple sclerosis. Lancet 1998;352:1498-1504

3. Jonhson KP, Brooks BR, Cohen JA, et al. Extended use of glatiramer acetrate (copaxone) is well tolerated and maintains its clinical effect on multiple sclerosis relapse rate and degree of disability. Neurology 1998;50:701-708.

4. Jacobs L, O'Malley J, Freeman A, Ekes R. Intramuscular interfereon beta 1-a for disease progression in relapsing multiple sclerosis. Ann Neurology 1996;39:285-294.

5. Kurtzke JF. Rating neurologic impairment in multiple sclerosis: na expanded disability status scale (EDSS). Neurology 1983;33:1444-1452.

6. Sipe JC, Knobler SL, Braheny GPA, Rice HS, Panitch MBA, Oldstone. A neurological rating scale (NRS) for use in multiple sclerosis. Neurology 1984;34:1368-1372.

7. Schumacher GA, Beebe G, Kilber RF, et al. Problems of experimental trials of theraoy in multiple sclerosis: report of the panel on evaluation of experimental trials in MS. Ann NY Acad Sci 1968;122:552-568.

8. Sharrack B, Hughes RAC. Clinical scale for multiple sclerosis. J Neurol Sci 1996;135:1-9.

9. Goodkin DE, Cookfair D, Wende K, et al., and Multiple Sclerosis Collaborative Research Group. Inter- and Intrarater scoring agreement using grades 1.0 to 3.5 of the Kurtzke Expanded Disability Status Scale (EDSS). Neurology 1992;42:859-863. 